\title{
Effect of magnetic field, thermal cycling and bending strain on critical current density of multifilamentary Bi-2223/Ag tape and fabrication of a pancake coil
}

\author{
T K DEY* and M K CHATTOPADHYAY \\ Cryogenic Engineering Centre, Indian Institute of Technology, Kharagpur 721 302, India \\ MS received 3 April 1997; revised 12 June 1997
}

\begin{abstract}
Multifilamentary HTSC tapes are important for their applications in various electrical devices. Powder-in-tube technique with improved optimized synthesis parameters is regarded as one of the most promising ways to prepare long-length multifilamentary $\mathrm{Bi}-2223 / \mathrm{Ag}$ tapes. Nevertheless, usefulness of such tapes depends on their electrical and mechanical properties. Critical current density of a $\mathrm{Bi}-2223 / \mathrm{Ag}$ tape with 37 filaments has been studied at $77 \mathrm{~K}$ with field, field orientation, thermal cycling and bending strain as parameters. Results have been discussed in light of various mechanisms and models. A small pancake coil has been fabricated out of the same tape and the test results presented.
\end{abstract}

Keywords. Multifilamentary Bi-2223/Ag tape; powder-in-tube technique; critical current density: thermal cycling; bending strain; pancake coil.

\section{Introduction}

Critical current density of superconductors is one of the most important properties which decides its potential for numerous electrical engineering applications. Salama et al (1992) reviewed different processes which are useful for synthesizing HTSC tapes with highly-textured microstructure and the ability to carry high current density. Efforts are now being made to prepare long-length mono and multifilamentary tapes of $\mathrm{Bi}-2223$ superconductors, using various techniques. Of these dip coat technique (Tomita et al 1996a), multilayer tape by continuous heat treatment (Hasegawa et al 1995), sequential processing (Ashworth et al 1995) and powder-in-tube process (Rosner et al 1992; Shukla et al 1992; Hautanen et al 1993; Gubser et al 1995; Minot et al 1995) appear to be promising. Superconducting and mechanical properties of high $T_{\mathrm{c}}$ superconductors are now known to be greatly improved when silver is used as the material to make the oxide-metal composite (Dou et al 1995). Apart from being a relatively simple technique, the powder-in-tube process is regarded as an attractive route for producing $\mathrm{Bi}-2212$ and $\mathrm{Bi}-2223$ multifilamentary tapes with the c-axis oriented microstructure leading to high $J_{\mathrm{c}}$ values. However, factors such as densification, grain alignment, heat treatment, grain growth, microstructural defects and phase assemblage play a significant role for the improvement in $J_{c}$ of Ag-cladded Bi-based superconducting tapes (Dou and Liu 1993).

In the present paper, we report our results on the influence of magnetic field and field orientation on the critical current density $\left(J_{c}\right)$ of multifiamentary (37 filaments) $\mathrm{Bi}-2223 / \mathrm{Ag}$ tape, which has been produced by powder-in-tube technique. The tape was also evaluated for critical current degradation due to thermal cycling and bending

* For correspondence 
strain. The characteristics of a small pancake coil fabricated from the same tape are also presented.

\section{Experimental}

The silver-sheathed $\mathrm{Bi}-2223$ tape with 37 filaments was prepared by powder-in-tube process and was supplied by $\mathrm{M} / \mathrm{s}$ Intermagnetics General Corporation, USA. Prereacted powders were initially optimized for high $T_{\mathrm{c}} 2223$ phase, using high-purity $(99.9 \%)$ oxides and carbonates of $\mathrm{Bi}, \mathrm{Pb}, \mathrm{Sr}, \mathrm{Ca}$ and $\mathrm{Cu}$ with the cation ratio $1 \cdot 8: 0 \cdot 4: 2 \cdot 0: 2 \cdot 2: 3 \cdot 0$. The powder was packed in silver tubes $(99 \cdot 9 \%$ pure $)$ and was drawn through a series of dies. Multiple lengths were cut from the drawn rod and were restacked into a second tube. The restacked rod was then rerolled to the final size $(0.2 \mathrm{~mm}$ thick and $100 \mathrm{~m}$ long) with intermediate annealing to avoid work hardening. The final heat treatment was performed at $870^{\circ} \mathrm{C}$ for $150 \mathrm{~h}$ under low oxygen partial pressure $(5-10 \%)$.

XRD pattern of the tape was taken after removing the silver sheath from one side, using $\mathrm{Cu} \mathrm{K} \alpha$ radiation as shown in figure 1 . XRD analysis was performed using a least square fit method, assuming a tetragonal unit cell. The XRD pattern shows strong $(00 \mathrm{~L})$ texturing of $\mathrm{Bi}-2223$ grains with $\mathrm{c}$-axis normal to the tape surface. Our XRD analysis could detect the existence of only one small peak for 2212 phase. This confirms that 2223 phase is the major superconducting phase present in the tape. The lattice parameters obtained, corresponding to 2223 phase, are $a=b=5.411 \AA$ and $c=37 \cdot 03 \AA$.

Superconducting transition temperature $\left(T_{\mathrm{c}}\right)$ and the critical current density $\left(J_{\mathrm{c}}\right)$ of the tape were measured using standard four-probe technique. Critical current density $\left(J_{c}\right)$ was measured as a function of magnetic field and also as a function of temperature between 77 and $64 \mathrm{~K}$. All the measurements were taken with the sample completely immersed in a liquid nitrogen bath. Temperature below $77 \mathrm{~K}$ was achieved and maintained by controlled pumping over $L N_{2}$ bath. A cryostat was designed for $J_{c}$ measurement, which incorporates provision for high current (100 amp) leads, as well as smooth rotation of the sample under magnetic field (angular resolution $1^{\circ}$ ). Sample length chosen was $50 \mathrm{~mm}$ and criterion adopted for $J_{\mathrm{c}}$ measurement was $1 \mu \mathrm{V} / \mathrm{cm}$.

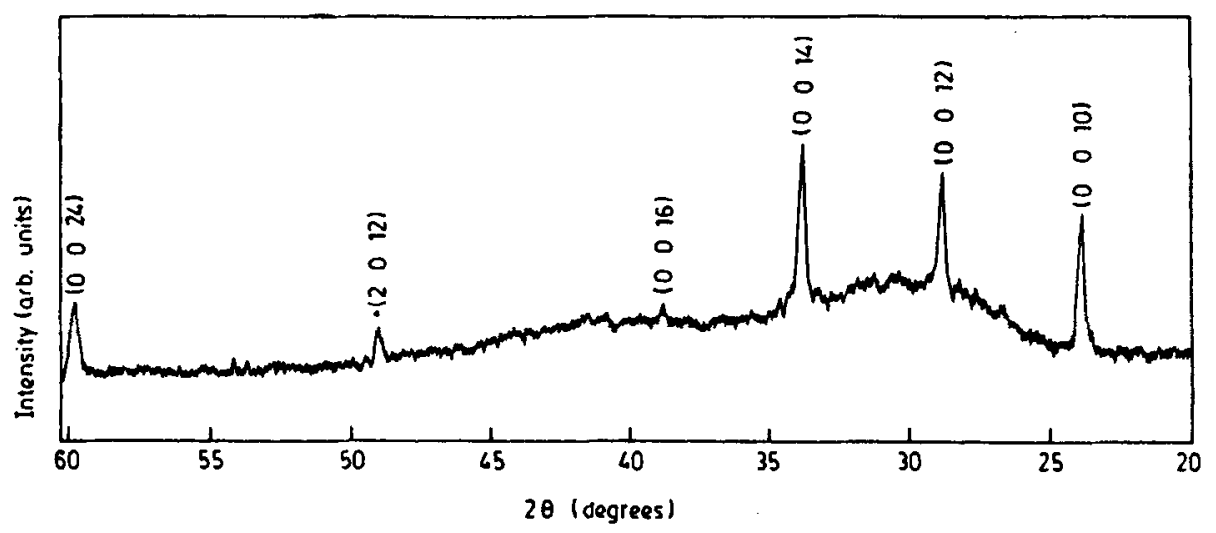

Figure 1. XRD pattern of Bi-2223/Ag multifilamentary tape. (-) corresponds to 2212 phase. 
Current and voltage leads were attached to the tape with indium. Voltage across the sample was measured with a resolution of $0 \cdot 1 \mu \mathrm{V}$ using a Keithley 181 Nanovoltmeter. A HP (model 6260B) Power Supply (10 V, $100 \mathrm{Amp}$ ) and a Ramp Programmer (AMI model 400) were used to generate the desired current ramp for $J_{c}$ measurement. The $I-V$ characteristics of the tape were plotted on an $\mathrm{X}-\mathrm{Y}$ recorder (Iakagawa model 302513). Temperature of the bath was monitored by a calibrated Si diode sensor coupled to Lakeshore model 330 Temperature Indicator-Controller. The external magnetic field was provided by an electromagnet. The overall uncertainty in the $J_{c}$ measurement was within $1 \%$.

\section{Results and discussion}

Temperature dependence of the resistance of the Ag-sheathed Bi-2223 tape, having 37 filaments is shown in figure 2. Sharp transition to superconducting state with $T_{\mathrm{c} 0}=107.4 \mathrm{~K}$ has been observed. Transition width $\left(\Delta T_{\mathrm{c}}\right)$ of the tape was close to $2 \mathrm{~K}$.

\subsection{Temperature and magnetic field dependence}

Critical current density $\left(J_{c}\right)$ of the tape as a function of temperature is shown in figure 3. Over the measured temperature range of $77-64 \mathrm{~K}$ in zero field, the critical current density increases approximately linearly with lowering of temperature. Variation of $J_{\mathrm{c}}$ of the tape at $77 \mathrm{~K}$ with magnetic field both parallel and perpendicular to c-axis is shown in figure 4a. The tape shows strong anisotropy in $J_{c}$, which increases with the increase of magnetic field (viz. $J_{\mathrm{c} \perp} / J_{\mathrm{c} \|} \cong 2$ at $0.1 \mathrm{~T}$ and $\sim 9$ at $0.3 T$ ). It is seen that $J_{\mathrm{c}}$ for magnetic field perpendicular to the broad face of the tape ( $B \| \mathrm{c}$-axis) is nearly an order of magnitude lower than that observed for $\mathrm{B} \perp \mathrm{c}$-axis. Further, decrease of $J_{\mathrm{c}}$ in magnetic field for $B \| \mathrm{c}$-axis is much more rapid $\left(J_{\mathrm{c}}\right.$ attains a very low value $\sim 275 \mathrm{~A} / \mathrm{cm}^{2}$ ) compared to the case when $B$ is perpendicular to the $c$-axis. Under zero field condition at 77 and $64 \mathrm{~K}$, the maximum $J_{\mathrm{c}}$ observed for the tape was $1.25 \times 10^{4} \mathrm{amp} / \mathrm{cm}^{2}$ and

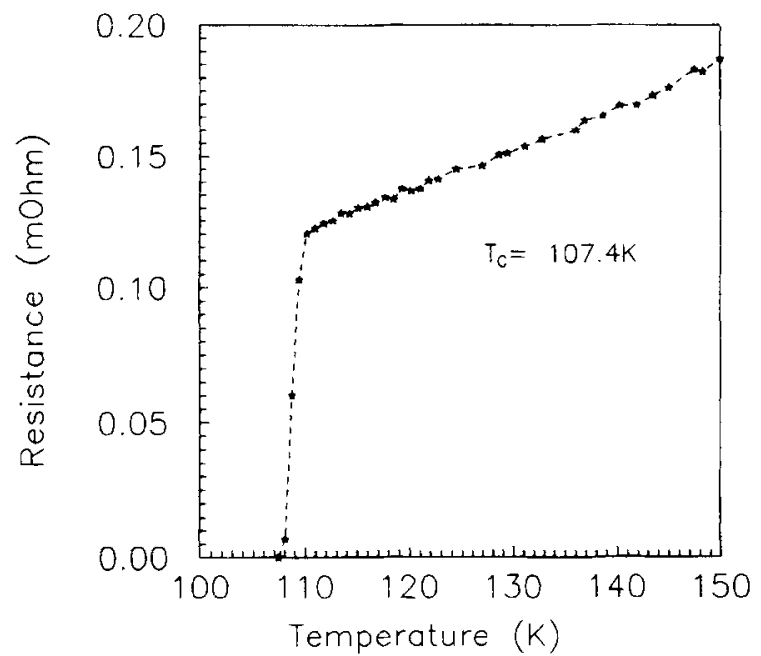

Figure 2. Resistance of $\mathrm{Bi}-2223 / \mathrm{Ag}$ multifilamentary tape as a function of temperature. 


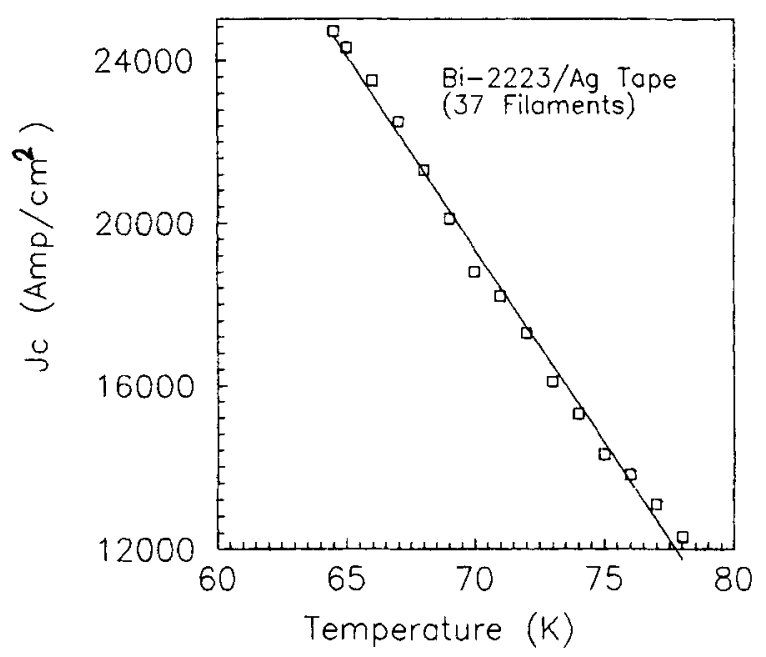

Figure 3. Critical current density of Bi-2223/Ag multifilamentary tape as a function of temperature at zero field.
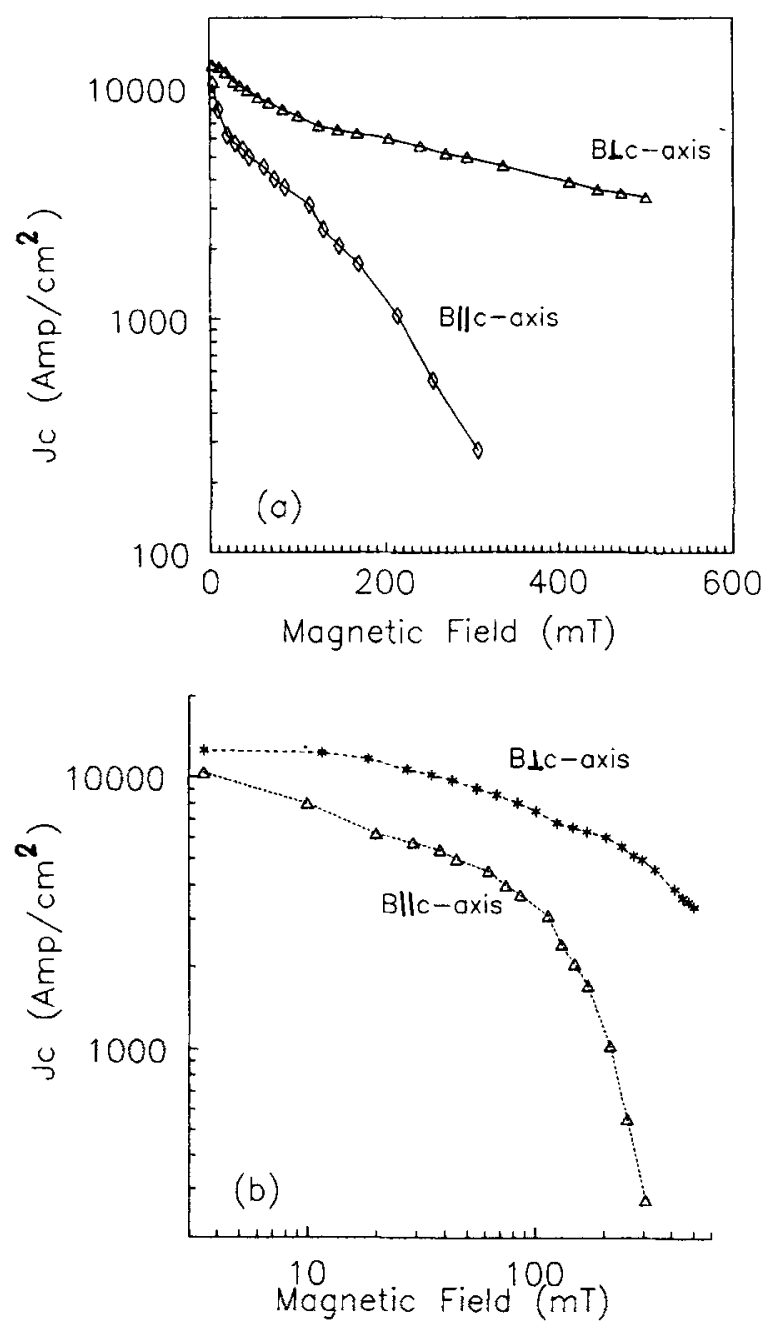

Figure 4. Magnetic field dependence of critical current density at $77 \mathrm{~K}$. (a) $\ln J_{\mathrm{c}}$ vs $B$ and (b) $\ln J_{\mathrm{c}}$ vs $\ln B$. 
$2.54 \times 10^{4} \mathrm{amp} / \mathrm{cm}^{2}$ respectively. Hysteretic behaviour of $J_{\mathrm{c}}$ of Ag-sheathed Bi 2223 tapes have been reported by Sato et al (1991), Grasso et al (1995) and others. Ries et al (1994), based on a model proposed by D'yachenko (1994), attributes this to the reversible shielding current and irreversible current due to pairing of Abrikosov vortices near the grain surface. It is important to note that we have not observed any hysteretic behaviour for our $\mathrm{Bi}-2223 / \mathrm{Ag}$ tape at $77 \mathrm{~K}$ in the field range between 0 and $0.6 \mathrm{~T}$.

Figure $4 \mathrm{~b}$ is the $\ln -\ln$ plot of the magnetic field dependence of $J_{\mathfrak{c}}$. The critical current density $\left(J_{c}\right)$ for our tape can be described by a power law of the type: $J_{c} \propto B^{-\alpha}$ up to about $100 \mathrm{mT}$, where the field independent component $\alpha$ has a value of $\sim 0.35$. This agrees with the other reports (Mawatari et al 1995b). For field higher than $100 \mathrm{mT}, J_{\text {c }}$ for $B \| \mathrm{c}$-axis shows an exponential dependence

$$
\left[\exp \left(-B / B_{0}\right)\right]
$$

where, $B_{0}$ is a field independent constant. It may be noted that in the low current region (viz. $<10^{2} \mathrm{amp} / \mathrm{cm}^{2}$ ), the effect of sharing current in the silver sheath can not be neglected and a correction for $J_{\mathrm{c}}$ may become necessary. Considering the Ag-sheathed superconducting tape as a parallel combination of the superconductor and the $\mathrm{Ag}$-sheath, the field dependence of $J_{c}$ can be obtained by subtracting the sharing current through the Ag sheath from the experimental value of $I_{\mathrm{c}}$. The $\left(J_{\mathrm{c}}-B\right)$ dependence observed in our tape, namely, power law dependence at low fields $(<100 \mathrm{mT})$ and exponential dependence for magnetic field $>100 \mathrm{mT}$ is consistent with the prediction of the brick wall model (Bulaevskii et al 1993), as well as collective pinning theory (Feigel'man and Vinokur 1990). From the measured data, the flux pinning force densities $\left(F_{\mathrm{p}}=J \times B\right)$ for the two orientations of magnetic field at $77 \mathrm{~K}$ have been computed and are shown in figure 5 as a function of magnetic field. As expected, flux pinning is substantially higher for $B \perp c$-axis. In the measured magnetic field range, a clear maximum in $F_{\mathrm{p}}$ evolves when $B \| \mathrm{c}$-axis, while for $B \perp \mathrm{c}$-axis $F_{\mathrm{p}}$ barely shows the sign of a maximum, but at a much higher field. However, in absence of $\left(J_{c}-B\right)$ data at different temperatures, it is rather difficult to come to any definite conclusion regarding the nature of scaling behaviour in the present case.

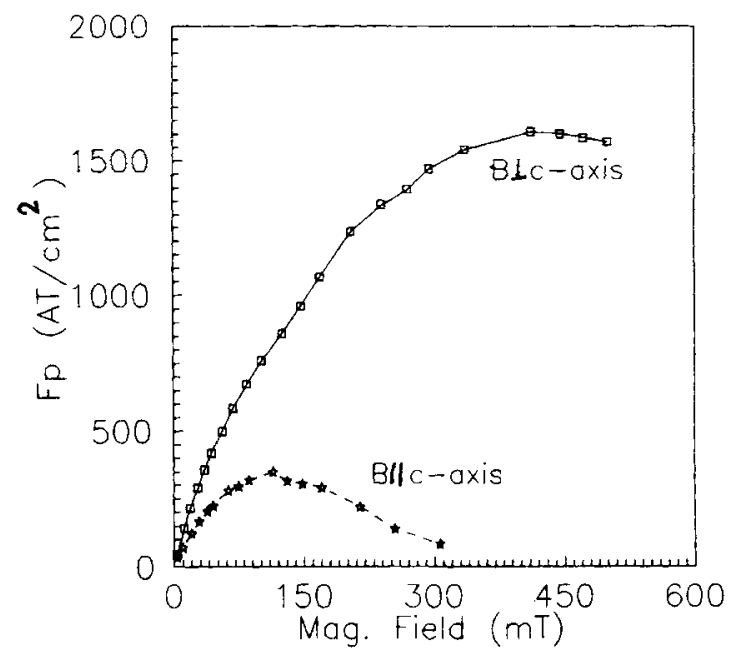

Figure 5. Magnetic field dependence of $F_{\mathrm{P}}$ at $77 \mathrm{~K}$. 

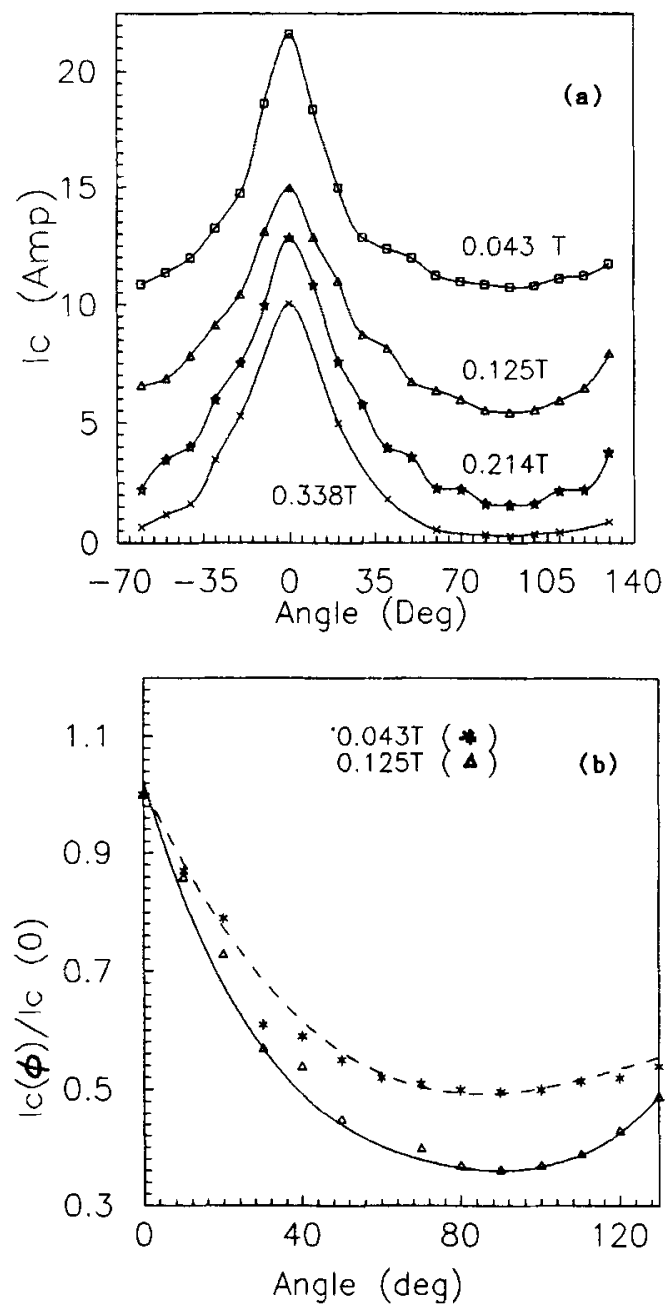

Figure 6. a. Angular dependence of $I_{\mathrm{c}}$ of Bi-2223/Ag multifilamentary tape at different applied fields and (b) $I_{c}(\phi) / I_{c}\left(0^{0}\right)$ vs $\phi$ for $B=0.043 T\left(^{*}\right)$ and $0.125 T(\Delta)$. The dashed and solid lines are the calculated values using the $T-T$ model for $B=0.043 T$ and $0.125 T$ respectively,

\subsection{Angular dependence}

Figure $6 \mathrm{a}$ shows the angular dependence of $J_{\mathrm{c}}$ of our Bi-2223/Ag tape for four different magnetic fields measured at $77 \mathrm{~K}$ and plotted as a function of the field tilt angle $(\phi)$. The angle $\phi$ has been defined as that between the applied field direction and the tape surface. It is seen from figure $6 \mathrm{a}$ that when the magnetic field is applied perpendicular to the broad face of the tape $\left(\phi=90^{\circ}\right), J_{\mathrm{c}}$ attains its lowest value. The highest value is observed for $B \perp \mathrm{c}$-axis $(\phi=0)$. Similar behaviour has been reported by Liebenberg et al (1992), $\mathrm{Hu}$ et al (1992) on Bi-2223 tapes as well as on thin films by Fukami et al (1993).

In the layered oxide superconductors the $\mathrm{CuO}_{2}$ layers and their vicinities are strongly superconducting and other layers are weakly superconducting. Accordingly, these materials may be considered to be made up of alternate stackings of strongly and 
weakly superconducting layers. The weakly superconducting layers act as natural pinning centres with considerably high pinning force (Tachiki and Takahashi 1989a). Assuming the presence of extrinsic planar pinning centres (effective for flux lines parallel to $c$-axis) in addition to the above mentioned intrinsic pinning centres, Tachiki and Takahashi (1989b) calculated the critical current density $\left(J_{c}\right)$ as a function of the direction of the applied external magnetic field. According to which, $J_{\mathrm{c}}(\phi)$ vs $\phi$ under the condition of $J$ perpendicular to $B$ is given by:

$$
\frac{J_{c}(\phi)}{J_{c}\left(0^{\circ}\right)}=\left[\frac{J_{c}\left(90^{\circ}\right)}{J_{c}\left(0^{\circ}\right)}\right] /|\sin (\phi)|^{1 / 2},
$$

where, $J_{c}\left(0^{\circ}\right)$ and $J_{c}\left(90^{\circ}\right)$ are the critical current densities in magnetic fields applied along the basal plane and parallel to the c-axis respectively. The dotted lines in figure $6 \mathrm{~b}$ show $J_{\mathrm{c}}(\phi) / J_{\mathrm{c}}\left(0^{\circ}\right)$ calculated using the above expression for different $\phi$ and for two different field strengths. It is seen that the $T-T$ model describes fairly well the angular dependence of $J_{\mathbf{c}}$ of silver-sheathed multifilamentary Bi-2223 tapes for low external fields. At higher fields, the deviations of the calculated values from the measured ones occur particularly in the low-angle regions. The cause for such deviations are not clearly understood.

\subsection{Thermal cycling}

A major problem reported for the powder in tube composite conductors has been the degradation of critical current density due to thermal cycling (van Sciver et al 1992; Jenkins et al 1993; Hilton and Hascieek 1995). Jenkins et al (1993) reported the occurrence of bloating (viz. opening up of Ag sheath) on thermal cycling which resulted in severe degradation of $J_{c}$. When bloating occurs $J_{c}$ degradation is expected due to considerable strain induced on the superconducting core. Other mechanisms, which may also cause $J_{c}$ degradation on thermal cycling are, differential thermal expansion within the HTSC tape (Ochiai et al 1991; Jenkins et al 1993) and pinholes penetrating the Ag sheath (Jenkins et al 1993). Therefore, to test the reliability of our Bi-2223/Ag tapes, $J_{c}$ of several pieces of the tape was measured at $77 \mathrm{~K}$ after repeated thermal cycling between room temperature and $77 \mathrm{~K}$. A total of 100 such thermal cycling were given to the tapes. Results obtained are shown in figure $7 . J_{\mathrm{c}}$ showed an initial nominal decrease of $\sim 4 \%$ after 10 cycles, beyond which it remained practically constant up to 100 cycles. It is important to note that no sign of bloating was noticed in our tapes, even after 100 thermal cycling between room temperature and $77 \mathrm{~K}$. However, when the end sections of the tape were not covered with indium, bloating of the silver sheath was observed in some samples after a few thermal cycling. In such cases, $J_{\mathrm{c}}$ showed a dramatic decrease, as is expected because of excessive strain on the superconducting filaments.

\subsection{Bending strain}

Depending upon the fracture strength, bending of tape results in micro cracks in the brittle ceramic filaments. Such cracks may be initiated by stress concentration around voids/pores, as well as by the inclusions from the secondary phase particles and 


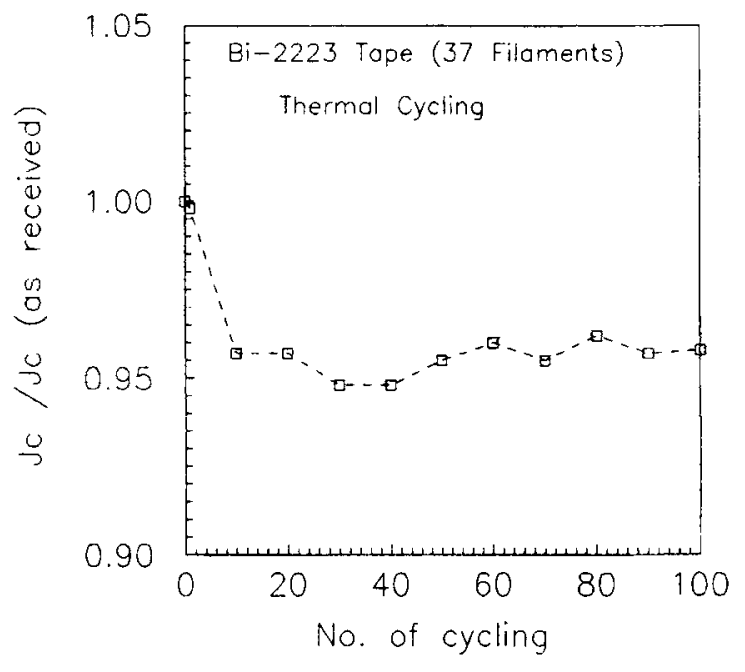

Figure 7. Normalized critical current density $\left(J_{c}\right)$ plotted as a function of number of thermal cycling between 300 and $77 \mathrm{~K}$ for $\mathrm{Bi}-2223 / \mathrm{Ag}$ sheathed tape.

agglomerates (Lawn 1993). This leads to an irreversible strain limit $\left(\varepsilon^{*}\right)$ above which permanent deterioration of $J_{c}$ occurs. Critical current density $\left(J_{c}\right)$ is generally reversible to its prestrain level below $\varepsilon^{*}$. Several reports on HTSC tapes (Sato et al 1991; Ekin et al 1992; Ochiai et al 1993; Sato 1993) indicate that strain degradation can be arrested either by decreasing the fill factor or by subdividing the core into multifilamentary geometries. Improved strain tolerance observed in multifilamentary tapes is thought to be due to limited crack propagation by increased Bi-2223/Ag interfaces (Osamura et al 1992).

Critical current degradation due to bending strain in our multifilamentary Bi2223/Ag tape was studied using a series of eleven non-metallic mandrills with bending diameters ranging from $90 \mathrm{~mm}$ to $12.9 \mathrm{~mm}$. Test sample was fixed on the surface of the mandrill with current and voltage leads attached by indium solder. Separation of the voltage leads was $10 \mathrm{~mm}$. Bending strain $(\varepsilon \%)$ on the superconducting core has been calculated from:

$$
\varepsilon=[t / 2 r] \times(100 \%),
$$

where, $r$ is the radius of bending and $t$ the thickness of the tape. Two sets of experiments were performed at 77 and $64 \mathrm{~K}$ under zero magnetic field. In the first set of experiments, $J_{\mathrm{c}}$ was measured with the tape in bent state on different mandrills (single bend), while for the second set the bent tapes were again straightened out prior to $J_{\mathrm{c}}$ measurements.

Figure 8a shows the normalized $J_{\mathrm{c}}\left[=J_{\mathrm{c}}(\varepsilon) / J_{\mathrm{c}}(0 \%)\right]$ as a function of the bending strain $(\varepsilon \%)$ for the tape at 77 and $64 \mathrm{~K}$. It is seen from figure $8 \mathrm{a}$ that $J_{\mathrm{c}}$ does not change significantly for bending strain up to about $0 \cdot 27 \%$, beyond which $J_{c}$ has been found to decrease irreversibly with increasing strain. At larger strain value $(\varepsilon>1 \cdot 1 \%)$, the critical current density shows a tendency of saturation. In our tape, $J_{c}$ retains half its zero strain value even at strain level of $0.8 \%$. Results on the present tape have been found to be consistent with the reported data on multifilamentary Bi-2223/Ag tapes having nearly same number of filaments (Osamura et al 1992; Carter et al 1995). In comparison to the data available on mono core $\mathrm{Bi}-2223 / \mathrm{Ag}$ tape (Osamura et al 1992), our tape displays 

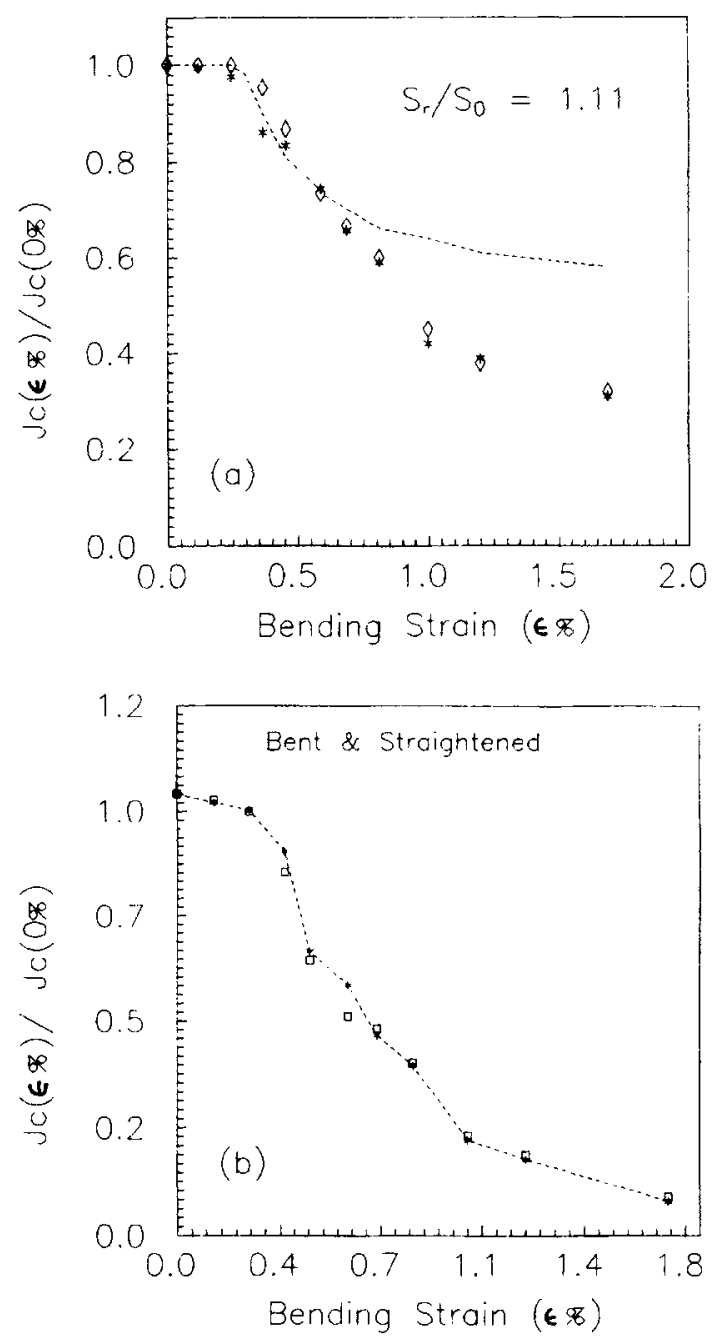

Figure 8. a. Bending strain $(\varepsilon \%)$ characteristics of the Bi-2223/Ag multifilamentary tape at $64 \mathrm{~K}(*)$ and $77 \mathrm{~K}(\diamond)$. Dashed line is the calculated values using the expression by Ot to $e t$ al $(1993)$ and $\mathbf{b} . J_{c}(\varepsilon \%) / J_{\mathbf{c}}(0 \%)$ vs bending strain $(\varepsilon \%)$ for tape bent to different diameters and then straightened prior to measurement.

a significant improvement in strain tolerance. Figure $8 \mathrm{~b}$ shows the results on the tapes which have been straightened after bending it once to different diameters on the mandrills. $J_{c}$ for such bent and straightened tapes not only displays beginning of degradation at low strain value $(\varepsilon=0 \cdot 14 \%)$, but also deteriorate faster with strain. Only $10 \%$ of the pre-strain $J_{c}$ value is retained when the tape is subjected to an initial bending strain of $1.8 \%$ and straightened before measurements.

A model describing the deterioration of critical current density due to fracture of superconducting filaments on the tensile side of the tape was proposed by Otto et al (1993), which in case of single bend is given by:

$$
I / I_{0}=\frac{S_{\mathrm{r}} \varepsilon^{*}}{2 S_{0} \varepsilon}+\frac{1}{2},
$$


where, $\varepsilon^{*}$ is the critical strain on the filaments under uniaxial tension, $2 S_{\mathrm{r}}$ the tape thickness, and $\left(S_{\mathrm{r}}-S_{0}\right)$ is the distance from the outside of the tape to the outermost filaments. The model assumes a uniform distribution of filaments in the region out to $S_{0}$ and that $I_{\mathrm{c}}$ of the filament drops to zero when the strain on the filament exceeds $\varepsilon^{*}$. The retained critical current density for an average critical strain of $0.27 \%$ for our tape has been calculated from the above equation and is shown in figure 8a. A fairly good agreement is obtained for strain up to $\varepsilon=0.7 \%$, beyond which deviations between the experimental and the predicted values are clearly seen. Otto et al (1993) also observed such deviations and argued that it originates due to the gradual current taper beyond the critical strain rather than the sudden decrease to zero critical current at the critical strain assumed in the model. Other reasons viz. silver work hardening and the loss of inter-plane symmetry in the properties might also contribute to the observed deviations at higher strain values.

\subsection{Fabrication of pancake coil}

Making of pancake coils have evolved due to the fact that it is easier to produce shorter lengths of tape than the longer ones, especially in the laboratory scale. Besides if one of the coils of the stacked pancake magnet breaks, it is much easier to replace the faulty coil than to replace the entire windings of the magnet (as in solenoidal coils). Preliminary studies reported by Okada et al (1995a, b), Tomita et al (1995b), Schwenterly et al (1995) and others on the performance of single and stacked pancake coils fabricated from $\mathrm{Bi}-2223$ tapes show promise for their use in high-field applications. We present here the fabrication details and the results obtained for a single pancake coil prepared using the present Bi-2223 tape $1.2 \mathrm{~m}$ long.

The coil was wound on a brass former (o.d. $=27 \mathrm{~mm}$ and i.d. $=23 \mathrm{~mm}$ ) with current leads extending perpendicular to the plane of the coil. Voltage taps separated by $1 \mathrm{~m}$ were soldered at the conductor ends. Number of turns in the coil was 10 . Teflon tape was cowound to provide interlayer insulation. After the winding of the tape, the coil was placed in a suitable casing and was impregnated with paraffin wax to improve its mechanical strength. In our design, the tape was exposed to a minimum bending radius of $14 \mathrm{~mm}$, which corresponds to $0.81 \%$ surface strain.

Voltage-current characteristics of the fabricated coil were measured by standard four-terminal method and critical currents were extracted using the electric field criterion of 10 and $100 \mu \mathrm{V} / \mathrm{m}$ over the temperature range from 64 to $77 \mathrm{~K}$ (figure 9). Index number $(n)$ was calculated from the measured $I_{\mathrm{c}}$ at two levels of electric field using the expression:

$$
n=\frac{\ln \left(E_{2} / E_{1}\right)}{\ln \left(I_{2} / I_{1}\right)},
$$

where, $E_{1}=10 \mu \mathrm{V} / \mathrm{m}$ and $E_{2}=100 \mu \mathrm{V} / \mathrm{m}$. The $n$ values of the fabricated coil ranged from 6.4 at $77 \mathrm{~K}$ to 6.7 at $64 \mathrm{~K}$. The generated magnetic field of the $\mathrm{Bi}-2223 / \mathrm{Ag}$ pancake coil was detected with a Hall sensor (M/s Lakeshore, USA) set at the centre of the coil. In zero background field, the coil generated a peak field of $2.5 \mathrm{mT}$ and $4.7 \mathrm{mT}$ at $77 \mathrm{~K}$ and $64 \mathrm{~K}$ respectively. This compares well with the results of similar coils (18.4 turns) reported by van Vo et al (1996) which generated a peak field of $5 \mathrm{mT}$. 


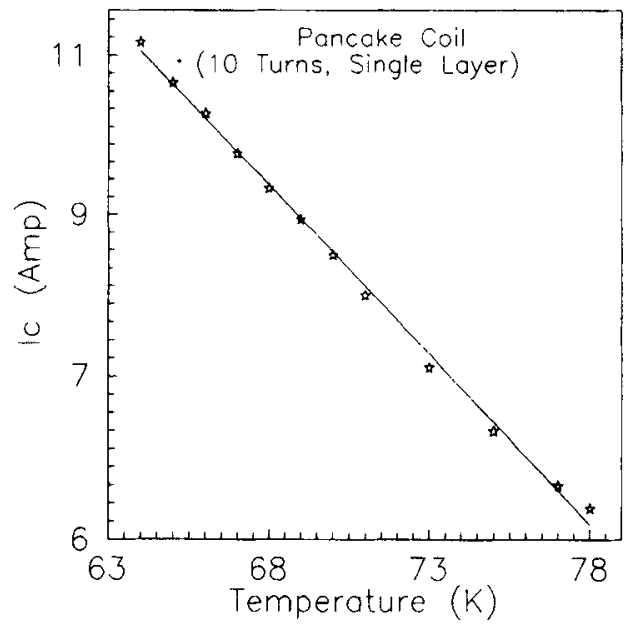

Figure 9. Critical current $\left(I_{\mathrm{c}}\right)$ of the single pancake coil as a function of temperature.

\section{Conclusion}

Silver sheathed $\mathrm{Bi}-2223$ tape with 37 filaments was prepared by a powder-in-tube method (supplied by $\mathrm{M} / \mathrm{s}$ Intermagnetics, USA). The tape shows a zero field $J_{\mathrm{c}}$ of $1.25 \times 10^{4} \mathrm{~A} / \mathrm{cm}^{2}$ at $77 \mathrm{~K} . J_{\mathrm{c}}$ of the tape displays strong anisotropy with the direction of applied magnetic field. Dependence of $J_{\mathrm{c}}$ on field orientation could be described fairly well by a model proposed by Tachiki and Takahashi (1995b). The tape shows good stability against repeated thermal cycling, as well as good strain tolerance to bending strain. $J_{\mathrm{c}}$ remains unaffected by the bending strain of $\sim 0.27 \%$ and maintains $\sim 50 \%$ of the prestrain $J_{c}$ at strain level of $0.8 \%$. A small 10 turns pancake coil has been fabricated and tested using $\sim 1 \mathrm{~m}$ long piece of the tape. The coil generates at $64 \mathrm{~K}$ a maximum field of $4.7 \mathrm{mT}$ carrying a current of $11.05 \mathrm{~A}$.

\section{Acknowledgements}

Authors are thankful to Dr $\mathrm{P}$ Halder at $\mathrm{M} / \mathrm{s}$ Intermagnetics General, USA for providing $\mathrm{Bi}-2223 / \mathrm{Ag}$ conductor for the present work. Technical help rendered by Mr D K Paul, Cryogenic Engineering Centre, is also gratefully acknowledged.

\section{References}

Ashworth S P, Glowacki B A, James M P, Garre R and Conti S 1995 IEEE Trans. Appl. Supercond. 51271 Bulaevskii L N, Daemen L L, Maley M P and Coulter J Y 1993 Phys. Rev. B48 13798

Carter W L, Ridey G N, Otto A, Parker D R, Christopherson C J, Masur L J and Buczek D 1995 IEEE Trans. Appl. Supercond. 51145

Dou S X and Liu H K 1993 Supercond. Sci.\& Technol. 6297

Dou S X, Guo Y C, Wang R K, Ionescu M, Liu H K, Babic E and Kusevic I 1995 IEEE Trans. Appl. Supercond. 51831

D'yachenko A I 1994 Physica C213 167

Ekin J W, Finnemore D K, Li Q, Tenbrink J and Carter W 1992 Appl. Phys. Letts 61858 
Fukami T, Lee D H, Panogopoulos C, Nishizaki T, Ichikawa F, Horie Y and Aomine T 1993 Physica C215 375

Feigel'man M V and Vinokur V M 1990 Phys. Rev. B41 8986

Grasso G. Hensel B, Jeremie A and Flükiger R 1995 IEEE Trans. Appl. Supercond. 51255

Gubser D U, Soulen R J, Datta T and Kirven D 1995 IEEE Trans. Appl. Supercond. 51302

Hasegawa T, Hikichi Y, Kumakura H, Kitaguchi H and Togano K 1995 Jap. J. Appl. Phys. 34 L1638

Hautanen K E, Oussena M and Cave J R 1993 Cryogenics 33326

Hilton D K and Hascieek Y S 1995 IEEE Trans. Appl. Supercond. 53478

Hu O Y, Liu H K and Dou S X 1992 Cryogenics 321048

Jenkins R G, Jones H, Belenli I, Yang M, Goringe M J and Grovenor C R M 1993 Cryogenics 3381

Lawn B 1993 in Fracture of brittle solids, Cambridge Solid State Series (UK: Cambridge University Press) 2nd Ed p. 307

Liebenberg D H, McIntyre P C, Cima M J and Francavilla T L 1992 Cryogenics 321066

Mawatari Y, Yamasaki H and Kosaka S 1995a IEEE Trans. Appl. Supercond. 51305

Mawatari Y, Yamasaki H, Kosaka S and Umeda M 1995b Cryogenics 35161

Minot M J, Buczek D, Gannon J J, Miles P K and Parker D R 1995 IEEE Trans. Appl. Supercond. 51246

Ochiai S, Hayashi K and Osamura K 1991 Cryogenics 31954

Ochiai S, Hayashi K and Osamura K 1993 Cryogenics 33976

Okada M, Tanaka K, Sato J, Awaji S and Watanabe K 1995a Jap. J. Appl. Phys. 344770

Okada M et al 1995b Jap. J. Appl. Phys. 34 L981

Osamura K, Ochiai S and Hayashi K 1992 Adv. Cryo. Engg. Mater. 38875

Otto A, Masur L J, Gannon J, Podtburg E, Daly D, Yurek G Y and Malozemoff A P 1993 IEEE Trans. Appl. Supercond. 3915

Ries G, Neumüller H W, Schmidt W and Struller C 1994 7th Int. workshop on critical currents in supercond. Alphach, Austria

Rosner C H, Walker M S, Halder P and Motowidlo L R 1992 Cryogenics 32940

Salama K. Selvamanickam V and Lee D F 1992 in Processing and properties of high $T_{\mathrm{c}}$ superconductors (ed.) S Jin (Singapore: World Scientific) 1 Chap. 5 p. 155

Sato K 1993 in Processing and properties of high $T_{c}$ superconductors (ed.) S Jin (Singapore: World Scientific) 1121

Sato K, Hikita T, Mukai H, Ueyama M, Kato T, Masuda T, Nagata M, Iwata K and Mitsui T 1991 IEEE Trans. Magn. 271231

Schwenterly S W et al 1995 IEEE Trans. Appl. Supercond. 5507

Shukla S R, Reddy Y S and Sharma R G 1992 Pramana-J. Phys. 38179

Tachiki M and Takahashi S 1989a Solid State Commun. 70291

Tachiki, M and Takahashi S 1989b Solid State Commun. 721083

Tomita N, Arai M, Yanagisawa E, Morimoto T, Kitaguchi H, Kumakura H, Togano K and Nomura K 1996a Cryogenics 36485

Tomita $\mathrm{N}$ et al 1995b IEEE Trans. Appl. Supercond. 520

Van Sciver S W, Hascieek Y S, Markiewicz W D, Motowidlo L R, Hazelton D R and Halder P 1992 Proc. 5th international superconductors symposium (Tokyo: Springer) p. 819

Van Vo N, Dou S X and Liu H K 1996 IEEE Trans. Appl. Supercond. 6102 